\title{
Synthesis of Tenoltrifluoroacetone Composite Doped with Terbium, Dysprosium and Europium Encapsulated in Silica Oxide Matrix
}

\author{
Yazmin Francisco', Dulce Medina ${ }^{1 *}$, Miguel Barron'1, Joan Reyes ${ }^{1}$, Pedro Adrian Martinez ${ }^{2}$, \\ Angel de Jesus Morales ${ }^{2}$, Elizabeth Garfias ${ }^{1}$ \\ ${ }^{1}$ Universidad Autónoma Metropolitana, Departamento de Materiales, Ciencias Básicas e Ingeniería, Reynosa Tamaulipas, México \\ ${ }^{2}$ Instituto Politécnico Nacional, ESIQIE, UPALM S/N, Mexico City, México \\ Email: *dyolotzin@azc.uam.mx
}

How to cite this paper: Francisco, Y., Medina, D., Barron, M., Reyes, J., Martinez, P.A., de Jesus Morales, A. and Garfias, E. (2020) Synthesis of Tenoltrifluoroacetone Composite Doped with Terbium, Dysprosium and Europium Encapsulated in Silica Oxide Matrix. Journal of Surface Engineered Materials and Advanced Technology, 10, 63-74.

https://doi.org/10.4236/jsemat.2020.104005

Received: September 24, 2020

Accepted: October 27, 2020

Published: October 30, 2020

Copyright $\odot 2020$ by author(s) and Scientific Research Publishing Inc. This work is licensed under the Creative Commons Attribution International License (CC BY 4.0).

http://creativecommons.org/licenses/by/4.0/

\section{(c) (i) Open Access}

\begin{abstract}
The synthesis of the thenoyltrifluoroacetone compound doped with terbium, dysprosium and europium encapsulated in a silica matrix (TTA:Tb:Dy:Eu@SiO${ }_{2}$ ) were performed by the sol-gel method. The precursors to obtain the vitreous phase $\left(\mathrm{SiO}_{2}\right)$ were: Tetraethylorthosilicate (TEOS, $\mathrm{C}_{8} \mathrm{H}_{2} \mathrm{O}_{4} \mathrm{Si}, 98 \%$, Aldrich), and ethyl alcohol $\left(\mathrm{CH}_{3} \mathrm{CH}_{2} \mathrm{OH}, 99.5 \%\right.$, Meyer), distilled water and $0.05 \mathrm{ml}$ of hydrochloric acid $(\mathrm{HCl}$, Meyer). The sample with molar ratio 20:80 TTA:Tb:Dy:Eu@SiO ${ }_{2}$ has the best emission intensity. Thermogravimetric analysis (TGA) shown that silica encapsulated samples decompose at lower temperatures than pure TTA:Tb:Dy:Eu luminescent material. Fourier Transform Infrared (IR-TF) shown the characteristic Si-O-Si bands that are presented at a wavelength of 1049, 853 and $440 \mathrm{~cm}^{-1}$ confirming that the luminescent material is encapsulated in a silica matrix, finally $\mathrm{X}$-ray diffraction (XRD) shown that TTA:Tb:Dy:Eu@SiO ${ }_{2}$ composite is amorphous.
\end{abstract}

\section{Keywords}

Synthesis, Luminescence, Composite, Sol-Gel, Terbium,

Dysprosium and Europium

\section{Introduction}

Luminescent materials are presented in a wide variety of materials such as inorganic crystals, glasses, ceramics and organic compounds [1] because the technological advance of luminescent materials has been growing since the applications that these have are diverse and are used in optoelectronics, medical appli- 
cations, textiles, and others applications. In this way, the study of organic binders doped with rare earth (such as $\beta$-diketones) is growing rapidly since it is possible to increase considerably the intensity of emission of compounds due to the so-called antenna effect [2].

Organic compounds of lanthanides have been widely reported to have desirable properties that show intense narrow-band emissions through efficient intermolecular energy transfer from the binding states to the central metal ions. The luminescence spectra are greatly intensified by the completion of lanthanide ions with organic ligands. These lanthanide ions form a stable crystalline complex with heterocyclic ligands, such as bipyridyl (bipy) and phenanthroline (phen10) [3] [4] [5]. On the other hand some projects have been reported to obtain low-cost luminescent material, using a silica matrix. Orozco [6], reported the synthesis and characterization of the luminescent and structural properties of zinc sulphide $(\mathrm{ZnS})$ powders, doped with the trivalent Europium ion $\left(\mathrm{Eu}^{3+}\right)$ and encapsulated in silicon dioxide $\left(\mathrm{SiO}_{2}\right)$, the luminescent properties of the powders were significantly improved when they were thermally treated, being the experiment nine with the ratio $\left[1: 2,1: 2,8 \%, 800^{\circ} \mathrm{C}\right]$ the one with the best result. Reyes et al. [7] obtained nanometric powders of $\mathrm{BaTiO}_{3}: \mathrm{Eu}^{3+}$ composite materials encapsulated in a $\mathrm{SiO}_{2}$ matrix they found that is possible to increase the intensity of light emission in the powders, when they are thermally treated at $200^{\circ} \mathrm{C}$ for $24 \mathrm{~h}$, according to the photoluminescence tests. Finally Y. Li et al. [8], synthesized $\mathrm{TNaGdF}_{4}: \mathrm{Yb}$, Er nanoparticles were encapsulated in $\mathrm{SiO}_{2}$ from the precursor TEOS by the sol-gel method. The light emission intensity of the $\mathrm{SiO}_{2}$ encapsulation was 5 times higher compared to the $\mathrm{NaGdF}_{4}: \mathrm{Yb}$ nanoparticles. Therefore, it is demonstrated that the coating of ceramic and organic particles in $\mathrm{SiO}_{2}$ improves the optical properties of the composite materials by increasing the light emission [9] [10] [11]. In this work, we improve this methodology in TTA:Tb:Dy:Eu organic powders.

\section{Methodology}

For the samples of the glass-ceramic materials, powders of TTA:Tb:Dy:Eu were used as precursors; which were previously obtained. To obtain the vitreous phase, Tetraethylorthosilicate (TEOS, $\mathrm{C}_{8} \mathrm{H}_{20} \mathrm{O}_{4} \mathrm{Si}, 98 \%$, Aldrich), and ethyl alcohol $\left(\mathrm{CH}_{3} \mathrm{CH}_{2} \mathrm{OH}, 99.5 \%\right.$, Meyer), distilled water and $0.05 \mathrm{ml}$ of hydrochloric acid ( $\mathrm{HCl}$, Meyer) were used. The vitreous solution was obtained by the hydrolysis and condensation of Tetraethylorthosilicate (TEOS) in ethanol and water as solvents, and adding acid as catalyst. The hydrolysis reaction occurs when Tetraethylorthosilicate (TEOS) and water are mixed in the ethyl alcohol, as it's shown in the following reaction:

$$
\mathrm{Si}\left(\mathrm{OC}_{2} \mathrm{H}_{5}\right)_{4}+4 \mathrm{H}_{2} \mathrm{O} \rightarrow \mathrm{Si}\left(\mathrm{OC}_{2} \mathrm{H}_{5}\right)_{4-4}(\mathrm{OH})_{4}+4 \mathrm{C}_{2} \mathrm{H}_{5} \mathrm{OH}
$$

$\mathrm{SiOH}$ groups are generated as an intermediate producer of the reaction. Complete hydrolysis of the alkoxide can result in $\mathrm{Si}(\mathrm{OH})_{4}$ salicylic acid, but does not occur. 
Synthesis of the composite TTA:Tb:Dy: $\mathrm{Eu} @ \mathrm{SiO}_{2}$

Five samples were made with different weight relations of TTA:Tb:Dy:Eu with respect to $\mathrm{SiO}_{2}$, as shown in Table 1 .

To do this, $0.05 \mathrm{~g}$ of TTA:Tb:Dy:Eu were weighed and placed in an agate mortar, the volume of $2 \mathrm{ml} \mathrm{SiO}_{2}$ was added until $8.90 \mathrm{ml} \mathrm{SiO}_{2}$ was reached and it was manually mixed with the pistil until a paste was formed and finally a composite powder of TTA:Tb:Dy:Eu@SiO ${ }_{2}$ was obtained. Once the TTA:Tb:Dy:Eu@SiO${ }_{2}$ composite is obtained, three samples with molar ratios of 5:95, 10:90 and 20:80 were placed in an oven at $88^{\circ} \mathrm{C}$ for 24 hours.

\section{Analysis of Results}

\subsection{Fourier Transform Infrared Spectroscopy (FT-IR)}

The IR spectrograms for the pure compound TTA:Tb:Dy:Eu, as well as the compound TTA: Tb:Dy:Eu@SiO ${ }_{2}$ were analyzed and the IR developed of the compound TTA:Tb:Dy:Eu@SiO ${ }_{2}$ with a molar ratio of 5:95, 10:90 and 20:80 were studied.

Figure 1 shows the spectrum of the control sample, which is TTA:Tb:Dy:Eu. A band in the range $3600-2900 \mathrm{~cm}^{-1}$ can be observed, which is attributed to the stretching vibrations hydroxyl group $(-\mathrm{OH})$ because there may be moisture in the sample. The bands TTA:Tb:Dy:Eu, likewise the bands of $\mathrm{C}_{-} \mathrm{CF}_{3}$ that indicate that the chelation of europium that predominates before the terbium and dysprosium was presented.

Figure 2 shows the IR spectrograms of theTTA:Tb:Dy:Eu@SiO ${ }_{2}$ composite at different molar ratios, where the TTA:Tb:Dy:Eu nanoparticles are encapsulated silica matrix. The presence of the bands in 3500 and $1650 \mathrm{~cm}^{-1}$ is observed, indicating that the silica contains a high amount of molecular water and hydroxyl. The band between $1610-1650 \mathrm{~cm}^{-1}$, is assigned to the vibrations of molecular water deformation $\delta \mathrm{OH}$ and results from the angular deformation $\mathrm{O}-\mathrm{H}$ in $\mathrm{H}_{2} \mathrm{O}$ $(\mathrm{H}-\mathrm{O}-\mathrm{H})$, so the band $1631 \mathrm{~cm}^{-1}$ can be attributed to residual ethanol. The bands at $1070 \mathrm{~cm}^{-1}$ are due to the asymmetric vas ( $\left.\mathrm{Si}-\mathrm{O}-\mathrm{Si}\right)$ stretching movement, in which the $\mathrm{O}$ atom moves back and forth along a line parallel to the axis joining the two $\mathrm{Si}$ atoms. The bands at $870 \mathrm{~cm}^{-1}$ correspond to the symmetrical stretch

Table 1. Experimental design of TTA:Tb:Dy:Eu@SiO ${ }_{2}$.

\begin{tabular}{cccccc}
\hline Exp. & \%TTA:Tb:Dy:Eu & \% $\mathrm{SiO}_{2}$ & $\begin{array}{c}\text { WTTA:Tb:Dy:Eu } \\
{[\mathrm{g}]}\end{array}$ & $\begin{array}{c}\mathrm{WSiO}_{2} \\
{[\mathrm{~g}]}\end{array}$ & $\begin{array}{c}\text { Vol. of solution } \mathrm{SiO}_{2} \\
{[\mathrm{ml}]}\end{array}$ \\
\hline $\mathbf{1}$ & 5 & 95 & 0.05 & 0.95 & 8.9039 \\
$\mathbf{2}$ & 10 & 90 & 0.1 & 0.90 & 8.4353 \\
$\mathbf{3}$ & 15 & 85 & 0.15 & 0.85 & 7.9667 \\
$\mathbf{4}$ & 20 & 80 & 0.20 & 0.80 & 7.4980 \\
$\mathbf{5}$ & 25 & 75 & 0.25 & 0.75 & 7.0294 \\
\hline
\end{tabular}




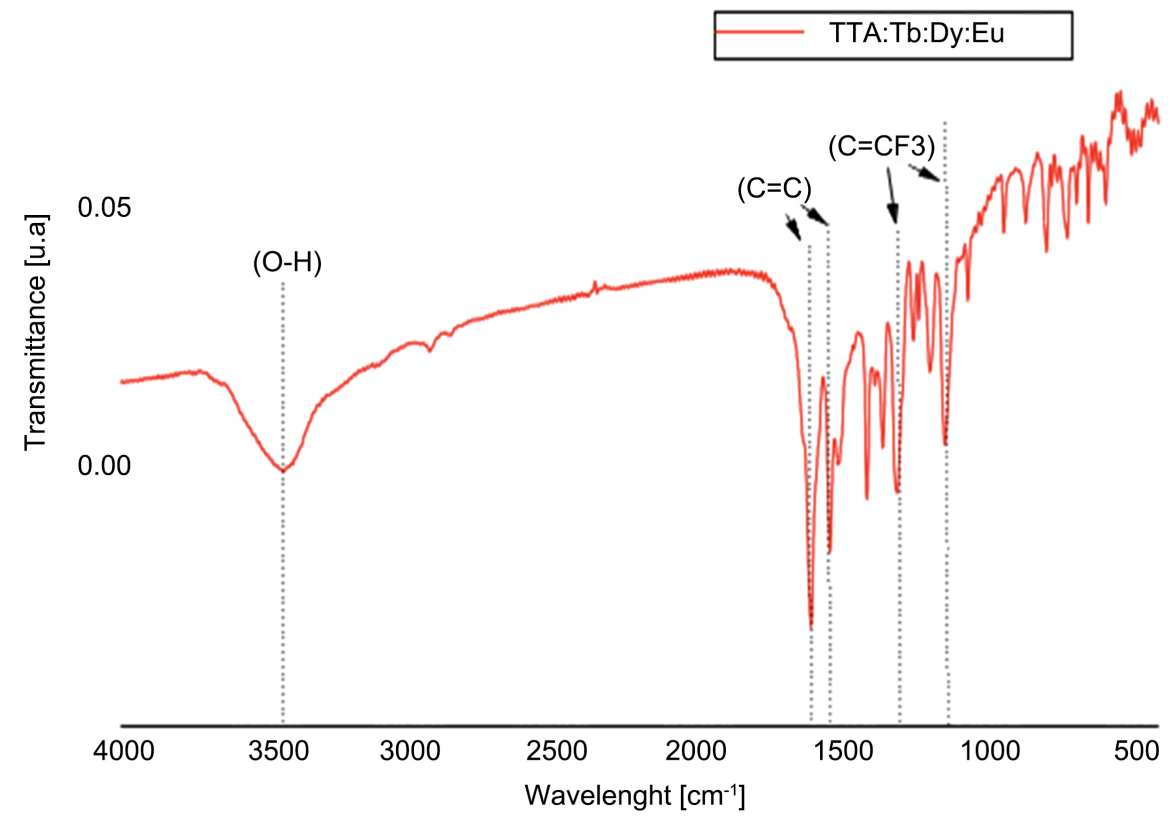

Figure 1. FT-IR Spectrum of TTA:Tb:Dy:Eu powder.

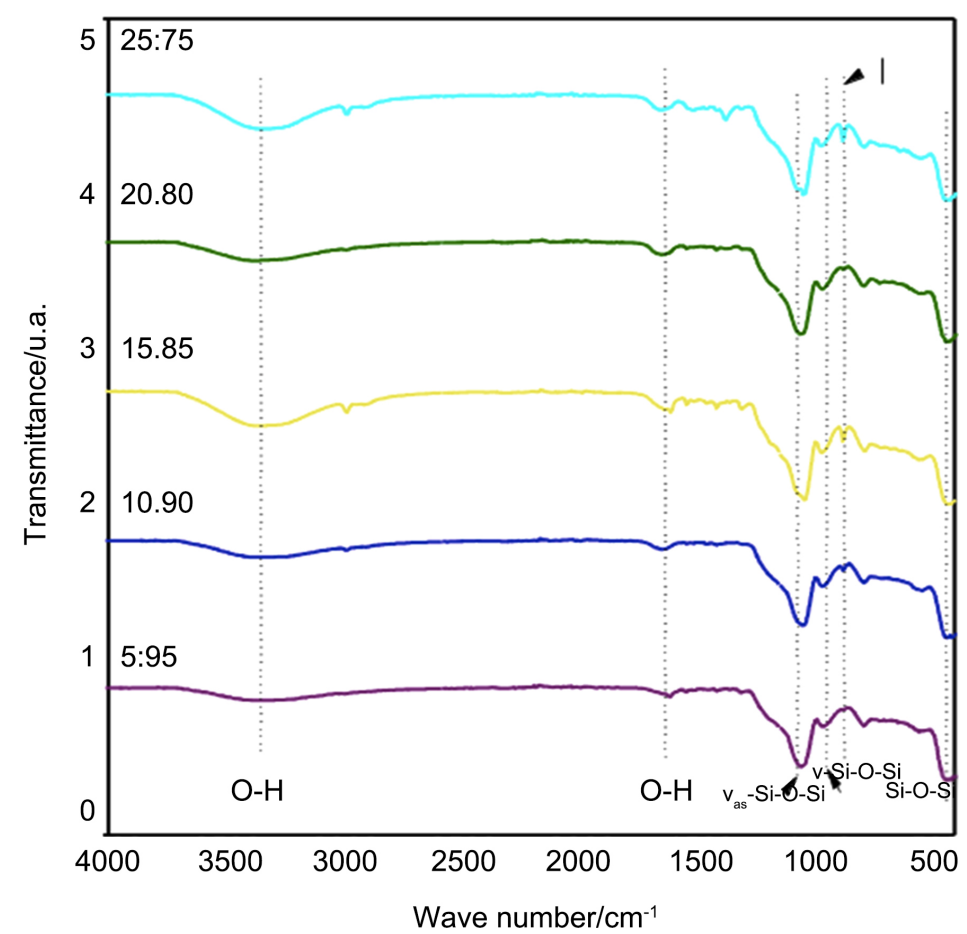

Figure 2. FTIR spectra of TTA:Tb:Dy:Eu@SiO 2 with different molar percent of $\mathrm{SiO}_{2}$ without thermal treatment.

of the $\mathrm{O}$ atom along the line bisecting the axis formed by the two $\mathrm{Si}$ atoms in siloxane bonds vs ( $\mathrm{Si}-\mathrm{O}-\mathrm{Si}$ ). The bands at $438 \mathrm{~cm}^{-1}$ are associated with rocking vibrations of the $\mathrm{O}$ atom around an axis that joins the two $\mathrm{Si}$ atoms in siloxane bonds ( $\mathrm{Si}-\mathrm{O}-\mathrm{Si}$ ). The band centered around $950 \mathrm{~cm}^{-1}$ is assigned to the stretching vibration of v-bonds ( $\mathrm{Si}-\mathrm{O}-\mathrm{Si})$. The spectra show that the amorphous silica 
matrix effectively encapsulates the luminescent material TTA:Tb:Dy:Eu since the characteristic bands of TTA:Tb:Dy:Eu are not present. As can be seen in Figure 2 , the structural bands between $1500-1550 \mathrm{~cm}^{-1}$ that are due to the stretching vibrations of the $\mathrm{C}=\mathrm{C}$ of the TTA disappear, only the characteristic spectrum of $\mathrm{SiO}_{2}$ is observed since the samples have a higher concentration of silica.

The spectra of FTIR TTA:Tb:Dy:Eu@SiO ${ }_{2}$ of the samples 5:95, 10:90 and 20:80 with heat treatment $88^{\circ} \mathrm{C}$ for 24 hours are also shown, as it can be seen in Figure 3. Where the spectra with molar ratios 5:95, 10:90 and 20:80 with heat treatment can be seen that the sample with molar ratio $20: 80$ has the characteristic spectrum of silica, while the sample 5:95 and 10:90 have the $\mathrm{C}_{-} \mathrm{CF}_{3}$ bonds since these bands are very defined. There are bands associated with the asymmetric vibration of Si-O-Si in the range of 1049 and $440 \mathrm{~cm}^{-1}$ these bands correspond to the silica matrix.

\subsection{Photoluminescence}

Excited emission spectra at $380 \mathrm{~nm}$ were performed, as well as the excitation spectra. First, the excitation spectrum is made to determine which is the best wavelength to excite the samples and obtain the highest emission intensity. The results that were obtained for the highest emission were in a length $\lambda=380 \mathrm{~nm}$, as can be seen in Figure 4. The compounds with respective molar ratios 5:95, 10:90, 15:82, 20:80 and 25:75 of TTA:Tb:Dy:Eu@SiO 2 are observed in this figure is can be observed that the peak proportion of 5:95 is the highest.

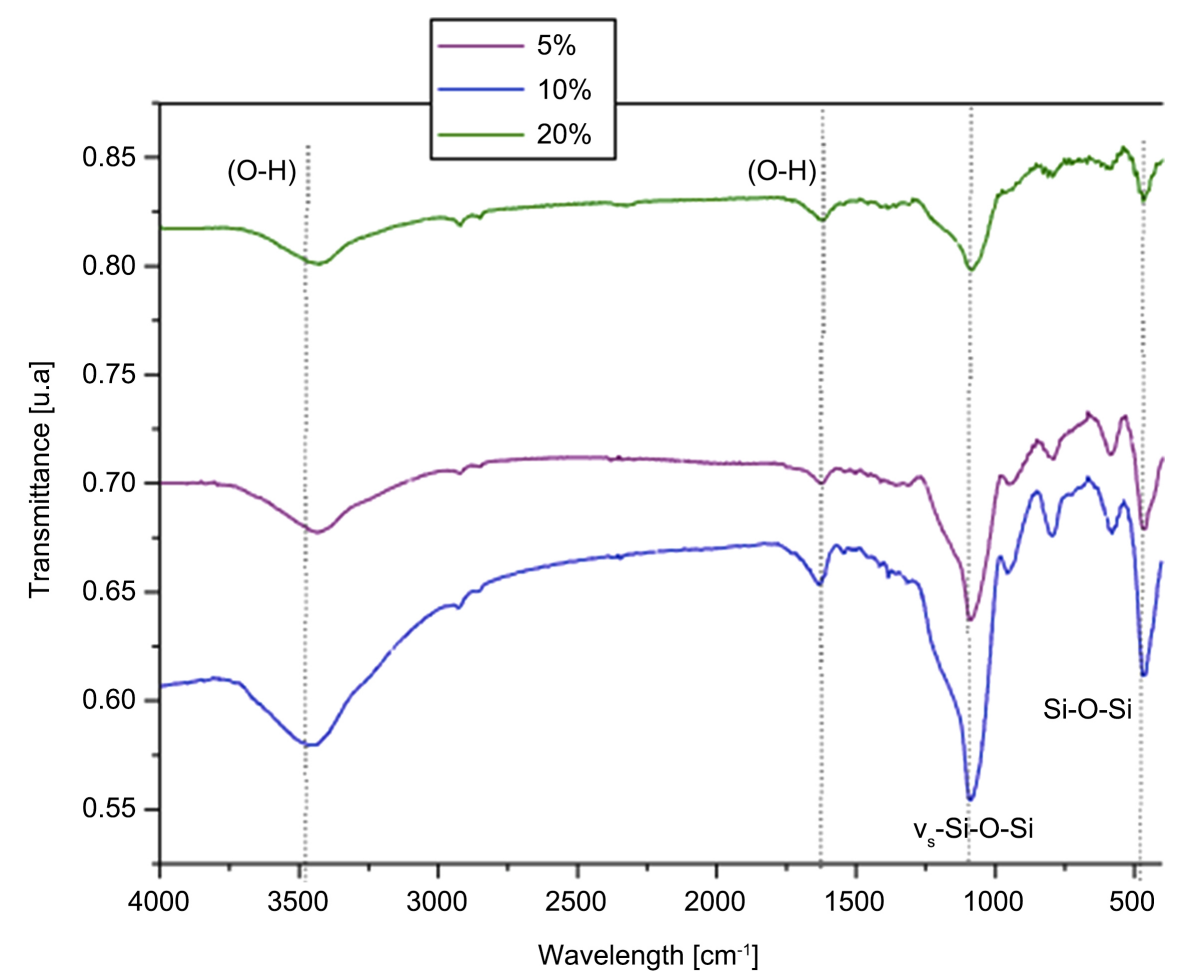

Figure 3. FT-IR spectra of samples 5TTA:Tb:Dy:Eu@95SiO ${ }_{2}$, 10TTA:Tb:Dy:Eu@90SiO 2 y 20TTA:Tb:Dy:Eu@80SiO ${ }_{2}$ with termal treatment at $88^{\circ} \mathrm{C}$ with 24 hours. 
In the case of the compound with molar ratio 20:80 purple color, two peaks are observed in $\lambda=306 \mathrm{~nm}$ and $\lambda=380 \mathrm{~nm}$, despite the fact that in that sample the highest is $\lambda=306$, the other samples do not present it therefore the peak of more intensity in the sample number five with the different molar ratios is the one found in $380 \mathrm{~nm}$. The emission bands, on the other hand as is shown in Figure 5 are characteristic of the $\mathrm{Eu}^{3+}$ transitions at $614.29,594.87,654.65$ and $705.44 \mathrm{~nm}$, with the highest emission correspond also to $5 \%$ of $\mathrm{SiO}_{2}$.

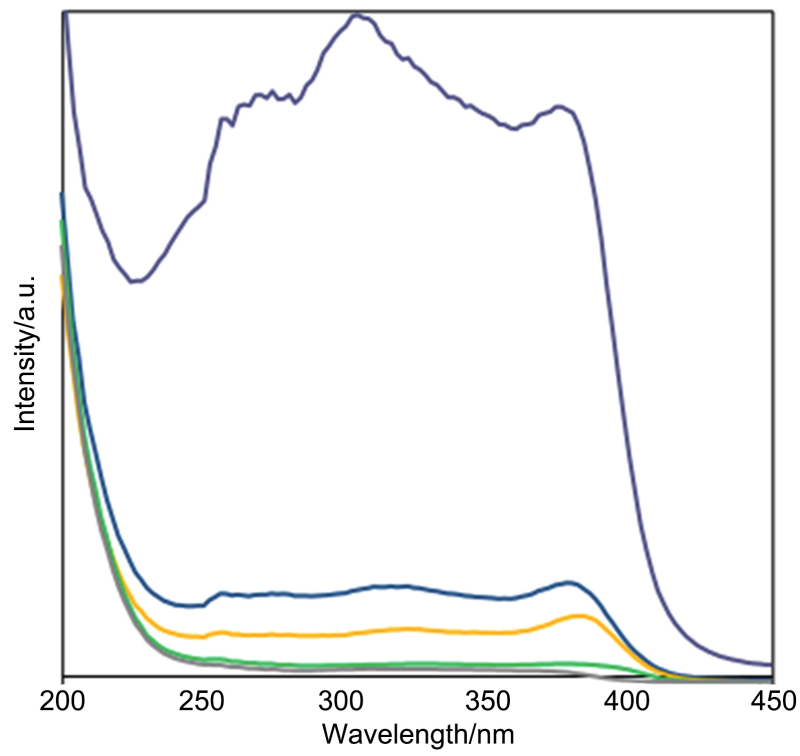

Figure 4. Excitation Spectrum of TTA:Tb:Dy:Eu@SiO ${ }_{2}$ with the different percent of $\mathrm{SiO}_{2}$ 5:95 (blue), 10:90 (yellow), 15:85 (green), 20:80 (purple), 25:75 (gray).

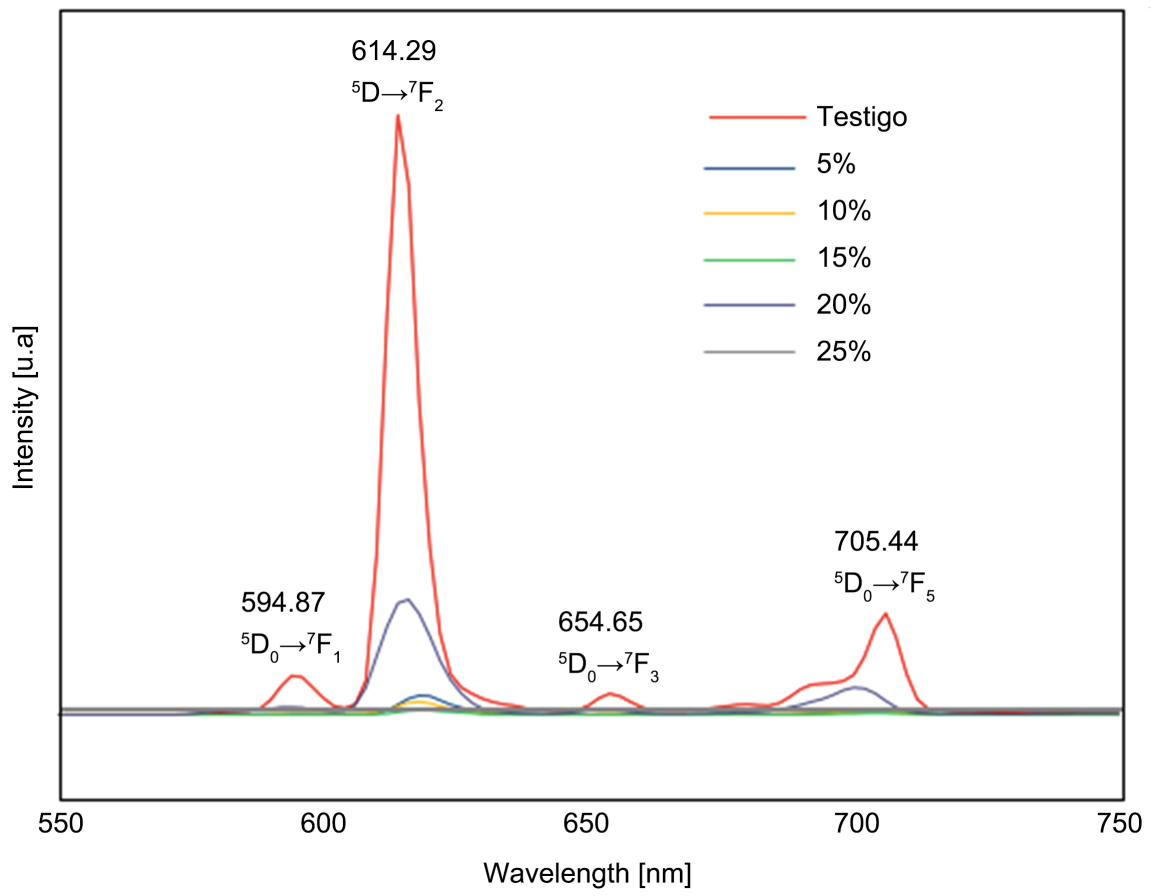

Figure 5. Spectra of emission TTA:Tb:Dy:Eu@SiO ${ }_{2}$, for the different molar ratios. 


\subsection{Chromaticity Diagram}

The chromaticity diagrams with their respective emission spectrum are shown below. The CIE chromatic coordinate of the compound TTA:Eu:Dy:Tb was a purity red-orange, this color is characteristic of the $\mathrm{Eu}^{3+}$ ion since it is the ion in the pure compound (TTA:Eu:Dy:Tb). The CIE chromaticity coordinate for composite TTA:Eu:Dy:Tb@SiO ${ }_{2}$ molar ratio 5:95, 10:90 and 20:80 in which you can note that for these two samples with 5\% TTA:Eu:Dy:Tb, 10\% TTA:Eu:Dy:Tb and 20\% TTA:Eu:Dy:Tb are those that maintain a red-orange purity equal to the control sample The sample containing 20\% TTA:Eu:Dy:Tb is closer. This can be verified in the following diagrams. The CIE chromaticity coordinates for the control sample (TTA:Eu:Dy:Tb) are shown, as well as the silica doped samples with the different molar ratios of TTA:Eu:Dy:Tb@SiO 2 . The displacement that the silica encapsulated samples have with respect to the pure sample is observed, where the molar ratio that is maintained in the purity of the red-orange region is the compound 20\% TTA:Eu:Dy:Tb@80\% $\mathrm{SiO}_{2}$, with these results it can be clearly seen that the samples that are in the red-orange region are the control (TTA:Eu:Dy:Tb), the compound that has 5\%, 10\% and $20 \%$ molar ratio TTA:Eu:Dy:Tb, while the samples with 15:85 and 25:75 molar ratio are displaced from the orange region. The values calculated CIE coordinates are shown in $\mathrm{Ta}$ ble 2 .

Figure 6 shows the comparison of the emission at the highest peaks of the TTA:Eu:Dy:Tb@SiO ${ }_{2}$ composite under an excitation of $380 \mathrm{~nm}$. It can be seen that the intensity of the silica encapsulated material increase when the composite sample has a molar ratio of 20:80, and the sample with a molar ratio of 25:75. Therefore, this diagram showed the compound with the highest luminous intensity TTA:Eu:Dy:Tb.

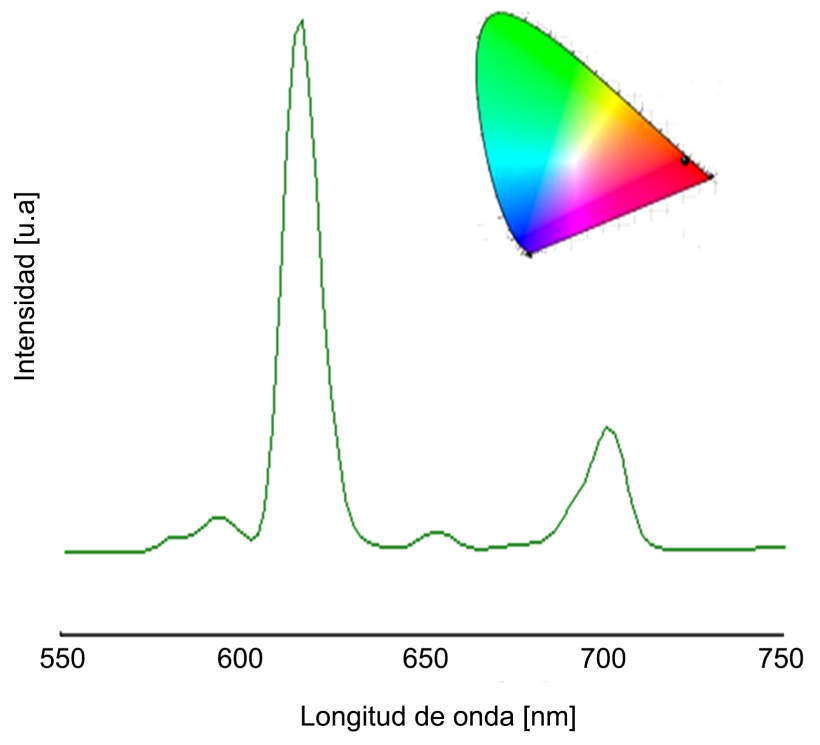

(a)

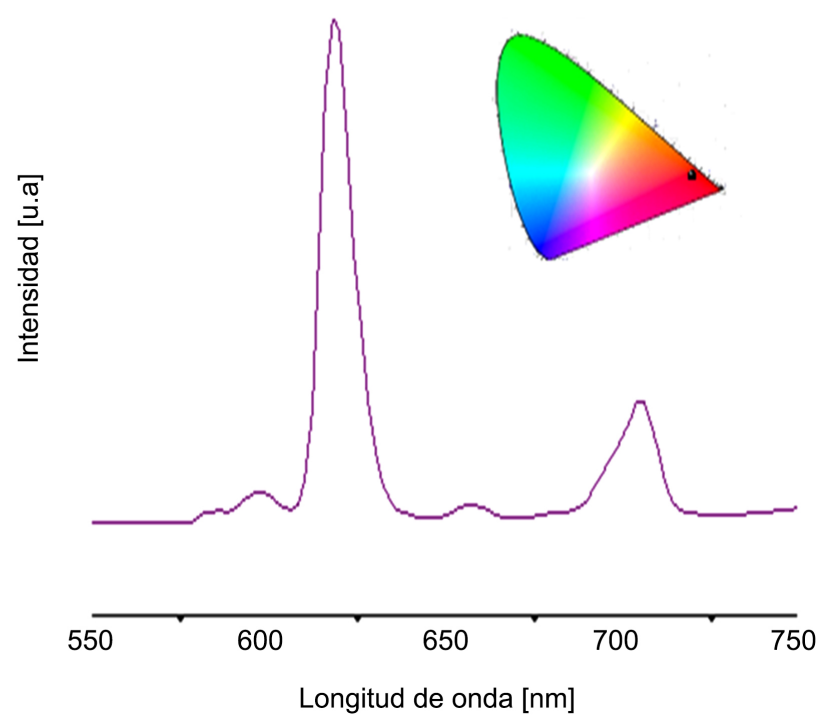

(b)

Figure 6. Spectra of emission TTA:Tb:Dy:Eu@SiO ${ }_{2}$, and CIE coordinator for (a) 20:80 and (b) 25:75. 


\subsection{X-Ray Diffraction Spectroscopy (XRD)}

The diffractograms presented in Figure 7 show the amorphous behavior of the material TTA:Eu:Dy:Tb@SiO ${ }_{2}$, as well as of TTA:Eu:Dy:Tb, with a crystal size in the order of nanometers. Likewise, a wide band can be observed that presents the composite Dy:Tb@SiO 2 in its different molar relations, where the shoulder grows with intensity in the samples that received thermal treatment at $88^{\circ} \mathrm{C}$, since when the temperature increases, the change of position of the peaks increases due to a possible change of phase. The uniform frames on the surface suggest that a homogeneous molecular base material was obtained by the strong covalent bond in the Si-O-Si networks that belong to a complex and large molecular system [9].

Table 2. CIE X, Y of the samples with different percentage of $\mathrm{SiO}_{2}$.

\begin{tabular}{|c|c|c|}
\hline Muestra & $\mathbf{x}$ & $y$ \\
\hline TTA:Eu:Dy:Tb & 0.66775392 & 0.32365108 \\
\hline TTA:Eu:Dy:Tb@SiO 2 5:95 & 0.63583116 & 0.32048406 \\
\hline TTA:Eu:Dy:Tb@SiO 2 10:90 & 0.63871604 & 0.326348 \\
\hline TTA:Eu:Dy:Tb@SiO 2 15:85 & 0.56552774 & 0.33406423 \\
\hline TTA:Eu:Dy:Tb@SiO $20: 80$ & 0.65539378 & 0.32666705 \\
\hline TTA:Eu:Dy:Tb@SiO 25:75 & 0.51643915 & 0.34470723 \\
\hline
\end{tabular}

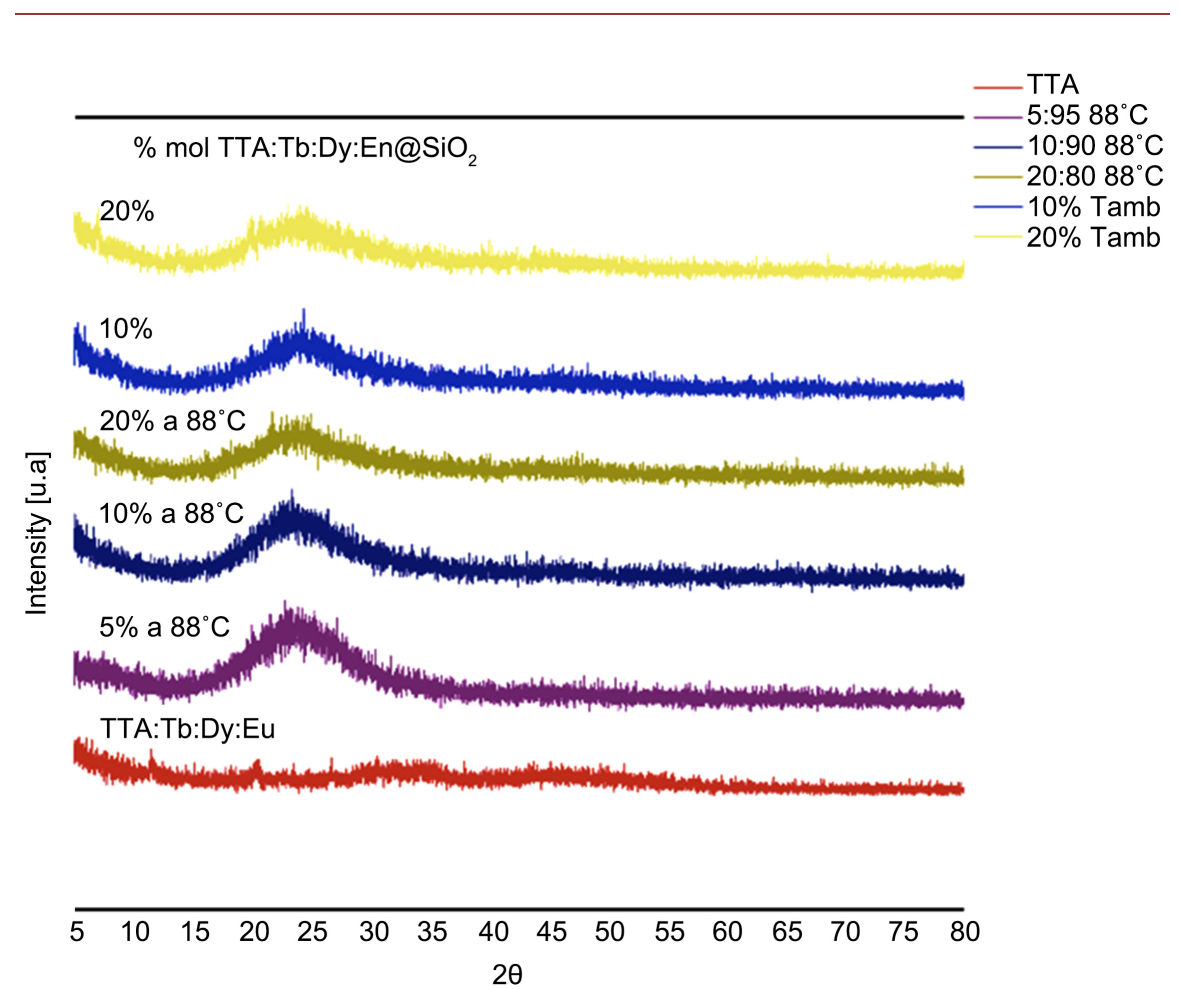

Figure 7. DRX spectra for different samples of TTA:Tb:Dy:Eu and TTA:Tb:Dy:Eu@SiO ${ }_{2}$, with different percentage and heat treatment. 


\subsection{Thermogravimetric Analysis (TGA)}

The thermogravimetric analysis of TTA:Eu:Dy:Tb shown in Figure 8 can be divided into four stages as the temperature increases, from $30^{\circ} \mathrm{C}$ to $100^{\circ} \mathrm{C}$, a peak decomposition center at $56^{\circ} \mathrm{C}$ and a weight loss of approximately $5 \%$ by weight is observed, which can be attributed to the release of surface moisture from TTA:Eu:Dy:Tb as TTA, you have another loss of $100^{\circ} \mathrm{C}$ to $231^{\circ} \mathrm{C}$ with $20 \%$ by weight. From $231^{\circ} \mathrm{C}$ to $400^{\circ} \mathrm{C}$ the weight loss was approximately $75 \%$ and a strong thermal absorption decomposition peak (center at $346^{\circ} \mathrm{C}$ ). From $350^{\circ} \mathrm{C}$, the weight loss was approximately $90 \%$ weight and a wide TTA:Eu:Dy:Tb was damaged.

Figure 9 shows the TGA curves for the composite TTA:Eu:Dy:Tb@SiO ${ }_{2}$ with a molar ratio 5:95 at 1) room temperature and 2) with heat treatment, differences in decomposition temperatures and mass losses. The compound with molar ratio of 5:95 shows that the weight loss occurred at lower temperatures. Once the colloidal solution of TTA:Eu:Dy:Tb and the $\mathrm{SiO}_{2}$ sun were mixed, you have a loss of $2 \%$ at a temperature of $31^{\circ} \mathrm{C}$ in this first stage is due to the losses of $\mathrm{OH}$ due to moisture and ethyl alcohol, you have a peak of decomposition at $310^{\circ} \mathrm{C}$ common loss of $83 \%$ weight. Meanwhile, the TGA curves for the composite TTA:Eu:Dy:Tb@SiO ${ }_{2}$ with a molar ratio 20:80 at 3) room temperature and 4) with heat treatment. The TTA:Eu:Dy:Tb@SiO${ }_{2}$ composites at room temperature shows a higher weight loss at $367^{\circ} \mathrm{C}$, while samples thermally treated at $88^{\circ} \mathrm{C}$ are the most weight loss are shown at $451^{\circ} \mathrm{C}$, therefore, it can be observed that when the sample heat treatment, you have a lower percentage of weight loss than with samples at room temperature, as well as the decomposition temperature increases for heat-treated samples.

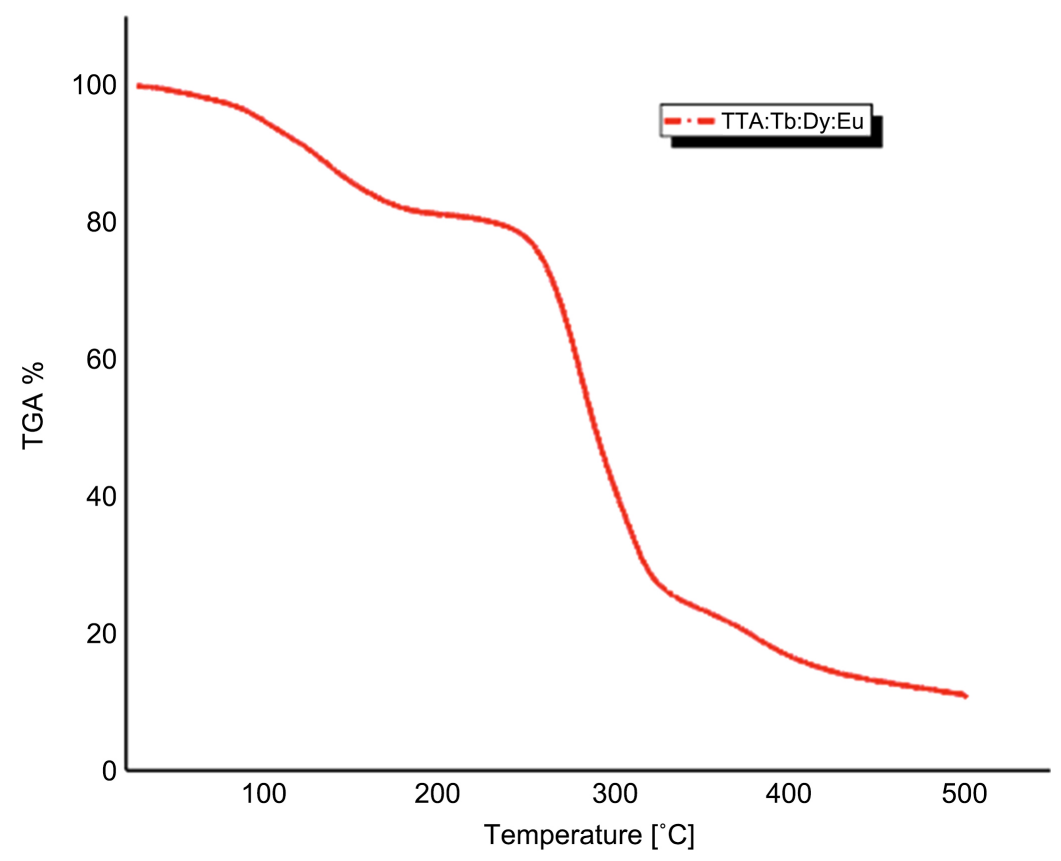

Figure 8. Thermogravimetric analysis of TTA:Eu:Dy:Tb sample. 

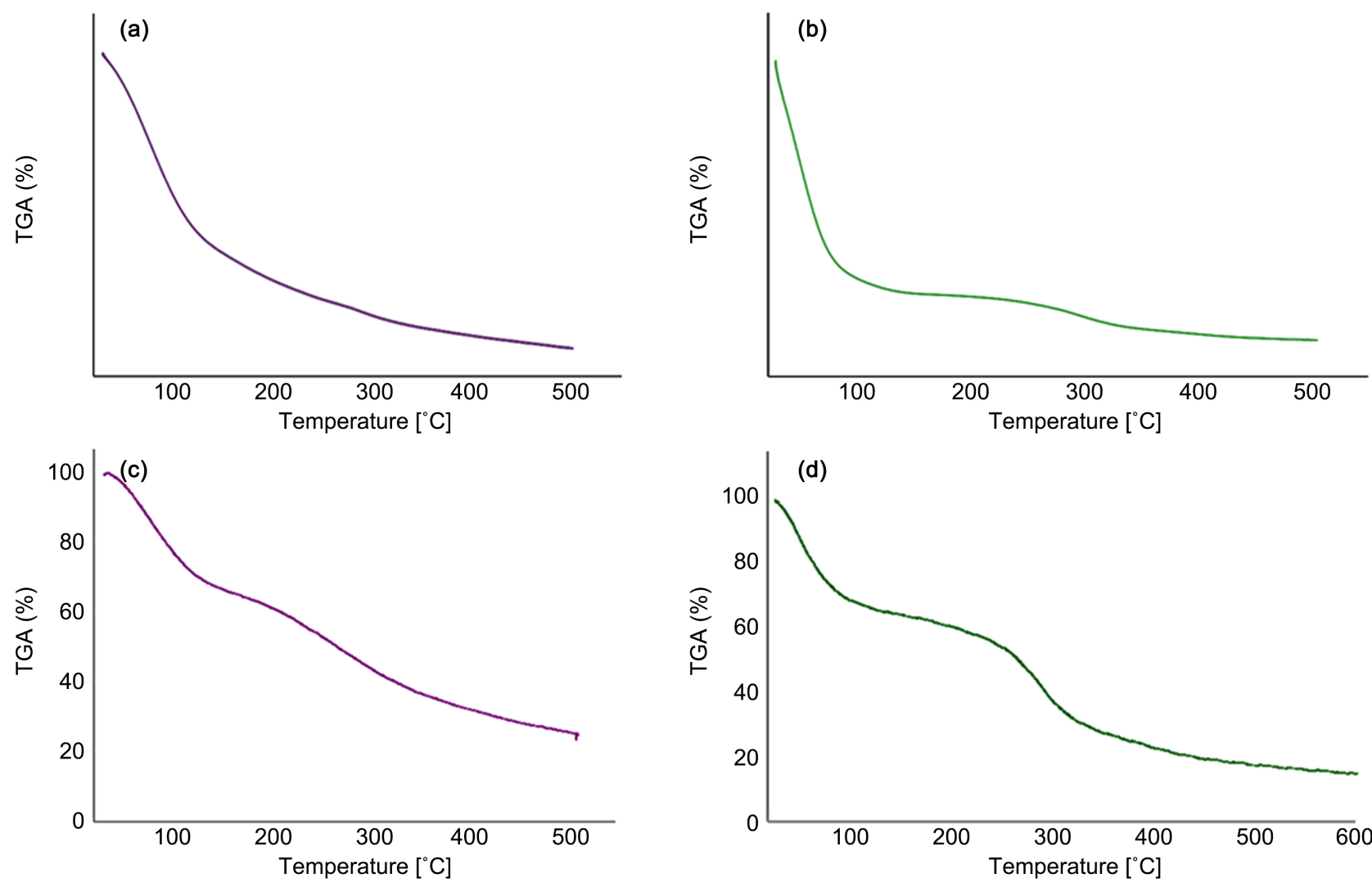

Figure 9. TGA studies for diferent percentage and heat treatment 5:95 at (a) room temperature (b) with heat treatment, and 20:80 (c) room temperature $(\mathrm{d})$ with heat treatment.

\section{Conclusions}

The TTA:Tb:Dy:Eu@SiO ${ }_{2}$ composite was synthesized by the sol-gel method, which presents a good luminescence. The amount of luminescent material at room temperature was increased from $0.7517 \mathrm{~g}$ of TTA:Tb:Dy:Eu to $9.8603 \mathrm{~g}$ of TTA:Tb:Dy:Eu@SiO ${ }_{2}$ by encapsulating it in a silica matrix without losing the red luminescence. By means of XRD, FL, FT-IR, TGA characterization techniques, structural and luminescent properties were determined.

The structure of the TTA:Tb:Dy:Eu@SiO ${ }_{2}$ composite was found to be amorphous at room temperature, as well as heat-treated to $88^{\circ} \mathrm{C}$. In the samples a wide band (shoulder) that has the highest intensity which is at 22 on the scale $2 \theta$ are observed.

By analyzing FT-IR in the silica-free TTA:Tb:Dy:Eu material, the presence of characteristic bands of TTA-related $\mathrm{C}=\mathrm{CF}_{3}$ links were confirmed. In the TTA:Tb:Dy:Eu@SiO ${ }_{2}$ composite the bands corresponding TTA disappear, keeping only the bands corresponding to $\mathrm{Si}-\mathrm{O}-\mathrm{Si}$ vibrations, corroborating the encapsulation of TTA: Tb:Dy:Eu in the $\mathrm{SiO}_{2}$ matrix. In both compounds, there are $\mathrm{OH}$ - group due to the incorporation of the ethanol solvent.

The photoluminescence analysis shows that the composite TTA:Tb:Dy:Eu@SiO with a molar ratio of $20 \%$ TTA:Tb:Dy:Eu and $80 \% \mathrm{SiO}_{2}$ obtains the highest luminescence than the rest of the composites due to the concentration of silica. 
The highest intensity was found at $\lambda=614 \mathrm{~nm}$ where the characteristic transitions ${ }^{5} \mathrm{D}_{0} \rightarrow{ }^{7} \mathrm{~F}_{2}$ of Eu${ }^{3+}$ appeared, confirming the color it emits is red-orange. The TGA analysis confirms a red-orange emission that tends to decrease with the silica concentration because the TTA:Tb:Dy:Eu@SiO ${ }_{2}$ composite decomposes at a lower temperature than when having the TTA:Tb:Dy:Eu material without silica.

\section{Acknowledgements}

This work was supported by CONACYT project 254280.

\section{Conflicts of Interest}

The authors declare no conflicts of interest regarding the publication of this paper.

\section{References}

[1] Azorin, N.J. (1993) Study of the Thermoluminescent and Optical Properties of the Main Dosimetric Materials, Thesis for the Degree of Doctor of Science (Physics). Autonomous Metropolitan University, Azcapotzalco Unit, Mexico.

[2] Medina, V.D.Y, Caldiño, U., Morales, R.A., Reyes, M.J., López, R.E., Escudero, R., Ruiz, G.R. and Morales, P.M.F. (2018) Synthesis of Luminescent TerbiumThenoyltriflouroacetone MOF Nanorods for Green Laser Application. Optical Materials, 87, 3-10.

[3] Meng, Q., Zhang, H., Wang, S., Fu, L., Zheng, Y. and Yang, K. (2000) Preparation and Characterization of Luminescent Thin Films Doped with Rare Earth $\left(\mathrm{Tb}^{3+}\right.$, $\mathrm{Eu}^{3+}$ ) Complexes Derived from a Sol-Gel Process. Material Letters, 45, 213-216. https://doi.org/10.1016/S0167-577X(00)00107-5

[4] Noriega, N.E., Carrillo, A., Castillo, S.J. and Mota, M.L. (2019) Production and Characterization of Non-Isocyanate Polyurethane/SiO $\mathrm{S}_{2}$ Films through a Sol-Gel Process for Thermal Insulation Applications. Polymer, 11, 1596. https://doi.org/10.3390/polym11101596

[5] Cabrialez, K.A. (2010) Obtaining 3C-SiC Nanoparticles by Microwave-Assisted Sol-Gel Synthesis: Thesis in Option to the Degree of Master of Science in Mechanical Engineering with Specialty in Materials. Autonomous University of Nuevo Leon, Mexico.

[6] Orozco, P.J.C. (2018) Synthesis and Characterization of $\mathrm{Zns:Eu}{ }^{3+}$ Passivated with $\mathrm{SiO}_{2}$ by the Polyol Technique. Terminal Project in Physical Engineering, Universidad Autónoma Metropolitana, Azcapotzalco Unit, Mexico.

[7] Reyes, M.J. (2013) Luminescent Properties of Powders of $\mathrm{ZnO}: \mathrm{Eu}^{3+} @ \mathrm{SiO}_{2}$ Obtained by the Solvothermal and Sol-Gel Methods. Thesis in Engineering Metallurgy and Materials, Instituto Politécnico Nacional, Mexico.

[8] Liu, Q., Wang, D.M., Li, Y.Y., Yan, M., Wei, Q. and Du, B. (2010) Synthesis and Luminescent Properties of $\mathrm{Eu}(\mathrm{TTA})_{3} \cdot 3 \mathrm{H}_{2} \mathrm{O}$ Nanocrystallines. Luminescence, 25, 307-310. https://doi.org/10.1002/bio.1150

[9] Guha, S. and Bojarczuk, N.A. (1998) Multicolored Light Emitters on Silicon Substrates. Applied Physics Letters, 73, 1487-1489. https://doi.org/10.1063/1.122181

[10] Zhang, X., Tang, J., Li, H., Wang, X., Wang, Y., Huang, L. and Belfiore, L. (2018) 
Red Light Emitting nano-PVP Fibers that Hybrid with Ag@SiO $\mathrm{O}_{2} \mathrm{Eu}(\mathrm{tta})_{3}$ phen-NPs by Electrostatic Spinning Method. Optical Materials, 78, 220-225.

https://doi.org/10.1016/j.optmat.2018.02.014

[11] Li, Y., Jiao, J., Yan, P., Liu, L., Wang, J., Wang, Y., Huang, L., Liu, J., Belfiore, L. and Tang, J. (2018) Synthesis and Optimizable Photoresponse for NaGdF4:Yb Nanocomplexes, Er@SiO ${ }_{2} @ E u(t t a)_{3}$ phen Structured Nucleus-Body. Scripta Materialia. 